\title{
Preoperative pre-albumin predicts prognosis of patients after gastrectomy for adenocarcinoma of esophagogastric junction
}

\author{
Wen-xiu $\mathrm{Han}^{\dagger}{ }^{\dagger}$, Zhang-ming Chen ${ }^{\dagger}$, Zhi-jian Wei and A-man Xu ${ }^{*}$
}

\begin{abstract}
Background: Adenocarcinoma of esophagogastric junction (AEG) was initially proposed in 1999 by Siewert. During recent decades, the incidence and prevalence of AEG were arising globally whereas the incidence of gastric cancer is gradually declining. Complete blood counting and liver function tests, as the routine examination of immune and nutritional status, were reported to be the predictors of overall survival (OS) in some tumors. However, little is known about the prognostic significance of these indexes in AEG patients. The purpose of this study was to assess the prediction of preoperative pre-albumin, hemoglobin, and prognostic nutritional index (PNI) for survival outcomes in AEG patients.

Methods: A retrospective cohort of 101 AEG patients followed by radical surgery was recruited between January and July 2010. Clinical and laboratory data were obtained and used to evaluate the predictive value through survival analysis. Receiver operating characteristic (ROC) curve analysis determined $200 \mathrm{mg} / \mathrm{L}, 120 \mathrm{~g} / \mathrm{L}$, $5 \mathrm{~cm}$, and 51 as the cutoff values of pre-albumin, hemoglobin, tumor size, and PNI, respectively.

Results: Univariate analysis revealed that AEG patients with hemoglobin $\geq 120 \mathrm{~g} / \mathrm{L}$, albumin $\geq 40 \mathrm{~g} / \mathrm{L}$, prealbumin $\geq 200 \mathrm{~g} / \mathrm{L}, \mathrm{PNI} \geq 51$, and tumor size $<5 \mathrm{~cm}$ had longer $\mathrm{OS}(P<0.05)$. Additionally, pre-albumin, tumor size, and TNM stage were demonstrated to be independent prognostic indicators by multivariate analysis with Cox regression, and the performance of pre-albumin for predicting OS in AEG patients was further identified by ROC curves $(P=0.006)$.
\end{abstract}

Conclusions: Preoperative pre-albumin was an independent prognostic factor, and a high level of prealbumin predicted longer OS in AEG patients.

Keywords: AEG, Gastric cancer, Pre-albumin, PNI, OS

\section{Background}

Gastric cancer (GC) was one of the most prevalent malignant diseases worldwide and ranked second for cancer deaths in 2013, especially in developing countries [1]. Adenocarcinoma of esophagogastric junction (AEG), as one of special malignant tumors due to their borderline location between the esophagus and stomach, was initially proposed in 1999 by Siewert

\footnotetext{
*Correspondence: xamhwx@163.com

${ }^{\dagger}$ Equal contributors

Department of Gastrointestinal Surgery, The First Affiliated Hospital of Anhui

Medical University, Hefei, Anhui Province 230032, China
}

[2]. During recent decades, the incidence and prevalence of AEG were arising globally whereas the incidence of gastric cancer is gradually declining [3-5]. Siewert et al. proposed that tumors at the location within $5 \mathrm{~cm}$ from the Z-line were defined as AEG independently and classified as three types, which have been widely adopted worldwide [6]. Recent epidemiological and clinical studies suggested that the prevalence, etiology, pathology, treatments, and outcomes of AEG were distinguishing obviously from tumors at other locations, even in the three types of AEG [7]. 
The competition between tumor aggression and body defense is crucial for prognosis of cancer-related overall survivals (OS). Among them, immune and nutritional status of patients with cancer had gradually become the focus in the field of cancer research nowadays [8], especially in patients with postoperative chemotherapy. Complete blood counting and liver function tests, as the routine examinations before surgery, were reported to be the predictors of OS in some tumors, such colorectal cancer, breast cancer, hepatocellular carcinoma, and GC [8-11]. Hemoglobin and albumin were the most common parameters to reflect the nutritional status, which of the lower level was demonstrated to be associated with poorer prognosis for patients with GC. Additionally, neutrophils to lymphocytes ratio (NLR) and platelets to lymphocytes ratio (PLR), as predictors of patients with GC, have been studied worldwide.

To our best knowledge, no studies had republished to access the prediction of these indexes for survival outcome in patients with AEG who underwent gastrectomy and chemotherapy. Here, the aim of this study was to research the clinical values of these parameters for prediction of OS in AEG patients.

\section{Methods}

\section{Patients}

The patients, who underwent radical open total or proximal gastrectomy for primary gastric cancer and were diagnosed as adenocarcinoma of esophagogastric junction based on postoperative pathology in the First Affiliated Hospital of Anhui Medical University (FAHAMU) from January to July 2010, were enrolled in this study retrospectively. All patients received the preoperative examinations of hemoglobin, albumin, pre-albumin, neutrophil, lymphocyte, and platelet. Additionally, complete clinicopathological characteristics including age, gender, tumor site, differentiation grade, tumor size, infiltration depth, lymph node metastasis, and distant metastasis were also collected. Patients who died within 30 days after surgery and received preoperative chemoradiotherapy were excluded from this study. Patients were also excluded if they underwent splenectomy or hepatectomy and had evidence of infections or were diagnosed with autoimmune diseases and multiple primary cancers. Finally, a total of 101 patients, who were followed up through telephones and outpatient visit up to September 2015, were enrolled in this study.

\section{Clinical and laboratory data collection}

All the details were collected from FAHAMU cancer database. The blood samples were gathered within 7 days before surgery to examine the hemoglobin, neutrophil, lymphocyte, platelet, albumin, globulin, and pre-albumin. According to the seventh edition of the American Joint Committee on Cancer (AJCC 2010) on tumor-node-metastasis (TNM) staging [12], the postoperative pathological stages were determined for patients with gastric cancer. Additionally, all AEG patients were classified into three types based on the AEG criteria recommended by Siewert (1998).

\section{Definition of prognostic nutritional index and cutoff values}

The prognostic nutritional index (PNI) was calculated using the following formula: $10 \times$ serum albumin $(\mathrm{g} / \mathrm{dL})$ $+0.005 \times$ total lymphocyte count $\left(\right.$ per $\mathrm{mm}^{3}$ ) [13]. The PNI cutoff points were selected by receiver operating characteristic (ROC) curve analysis for the prediction of survival outcomes based on data from the whole cohort. Finally, using the Youden index [maximum (sensitivity + specificity -1)] [14], we determined that the recommended cutoff value was 51 [sensitivity, 69.2; specificity, 59.2; area under the curve (AUC), $0.615 ; P=0.046]$. And according to the recommended cutoff value, patients were divided into groups as follows: low-PNI group (PNI $<51$ ) and high-PNI group (PNI $\geq 51$ ).

The recommended cutoff values for preoperative hemoglobin and pre-albumin were decided using ROC curve analysis based on the most prominent points on the ROC curves and defined as $120 \mathrm{~g} / \mathrm{L}$ [sensitivity, 51.9; specificity, 71.4; area under the curve (AUC), 0.621; $P=0.037$ ] and $200 \mathrm{mg} / \mathrm{L}$ [sensitivity, 44.0; specificity, 88.0; area under the curve (AUC), 0.659; $P=0.006]$.

\section{Statistical analysis}

Statistical Package for the Social Sciences, version 21.0 (SPSS, IBM, Chicago, IL, USA), was used for all statistical analysis, and differences at $P$ value $<0.05$ were considered to be significant in all statistical analysis. The ROC curves were constructed to determine the cutoff values of hemoglobin, pre-albumin, PNI, and tumor size. Additionally, the relationships between associated factors and overall survival were analyzed through the KaplanMeier method and compared by the log-rank test, respectively. Moreover, multivariate analysis was performed based on the univariate analysis with $P<0.05$ to evaluate the most valuable predictor of survival outcomes.

\section{Results}

\section{Baseline of patients' characteristics}

The clinicopathological characteristics of the 101 AEG patients and their relationships with overall survival were summarized in Table 1 . Overall, 80 (79.2 \%) patients were males and $21(20.8 \%)$ were females. The mean age of patients was 65 years old (range, 43-82). The median follow-up period was 51 months (range, 
Table 1 Clinical and laboratory characteristics of 101 AEG patients associated with OS

\begin{tabular}{llll}
\hline Patient-related factors & No. of patients (\%) $\begin{array}{l}\text { OS (months) } \\
{[\text { mean }(95 \% \mathrm{Cl})]}\end{array}$ & $P$ values \\
\hline $\begin{array}{l}\text { Gender } \\
\quad \text { Male }\end{array}$ & $80(79.2)$ & $42.3(36.9-47.7)$ & 0.343 \\
$\quad$ Female & $21(20.8)$ & $48.5(39.4-57.7)$ & \\
Age (years) & & & \\
$\quad<60$ & $23(22.8)$ & $44.5(34.9-54.1)$ & 0.976 \\
$\geq 60$ & $78(77.2)$ & $43.3(38.9-48.3)$ & \\
BMl (kg/m $\left.{ }^{2}\right)$ & & & \\
$<18.5$ & $9(8.9)$ & $31.7(18.8-14.6)$ & 0.180 \\
$\geq 18.5$ and $<25$ & $71(70.3)$ & $45.5(40.0-51.1)$ & \\
$\geq 25$ & $21(20.8)$ & $41.4(30.7-52.0)$ & \\
Hemoglobin (g/L) & & & \\
$<120$ & $41(40.6)$ & $38.4(31.4-45.4)$ & $0.029^{*}$ \\
$\geq 120$ & $60(59.4)$ & $44.6(41.0-53.4)$ &
\end{tabular}

Albumin (g/L)

$<40$
$\geq 40$
Pre-albumin (g/L)

$\begin{array}{ll}<200 & 29(28.7) \\ \geq 200 & 72(71.3) \\ \mathrm{PNI} & \\ <51 & 54(53.5) \\ \geq 51 & 47(46.5)\end{array}$

Tumor size $(\mathrm{cm})$

$<5$

$35.7(26.4-44.9) \quad 0.036^{*}$ $46.0(38.9-48.3)$

$31.5(23.6-39.3) \quad<0.001^{*}$

$48.0(43.0-53.8)$

$37.6(31.0-44.2) \quad 0.008^{*}$

$50.5(44.3-56.7)$

$48.3(42.5-54.1)$

$\geq 5$

$37(36.6)$

Differentiation grade

$$
\text { Poor }
$$

Well

$32(31.7)$

Tumor location

$\begin{array}{ll}\text { Siewert II } & 6(5.9) \\ \text { Siewert III } & 95(5.1) \\ \text { T stage } & \\ \text { T1, T2 } & 18(17.8) \\ \text { T3, T4 } & 83(82.2)\end{array}$

$\mathrm{N}$ stage

$\begin{array}{ll}\text { N0 } & 41(40.6) \\ \text { N1 } & 43(42.6) \\ \text { N2 } & 16(15.8) \\ \text { N3 } & 1(1.0)\end{array}$

TNM stage
Table 1 Clinical and laboratory characteristics of 101 AEG patients associated with OS (Continued)

\begin{tabular}{|c|c|c|c|}
\hline Surgical type & & & \\
\hline Total gastrectomy & $6(5.9)$ & $57.0(42.7-71.3)$ & 0.131 \\
\hline Proximal gastrectomy & $95(94.1)$ & $42.7(37.8-47.6)$ & \\
\hline
\end{tabular}

1.5-66), and there were $52(51.5 \%)$ cases confirmed as dead at the last follow-up.

\section{Univariate analysis of prognostic factors}

Among all 101 AEG patients, there are no significant differences observed in gender, age, BMI, and tumor location associated with OS. However, according to the univariate analysis, AEG patients with hemoglobin $\geq 120 \mathrm{~g} / \mathrm{L}$, albumin $\geq 40 \mathrm{~g} / \mathrm{L}$, pre-albumin $\geq 200 \mathrm{~g} / \mathrm{L}$, tumor size $<5 \mathrm{~cm}$, PNI $\geq 51$, and well differentiation grade had longer OS $(P<0.05)$, and this was consistent with an earlier T stage, $\mathrm{N}$ stage, or TNM stage $(P<0.01)$. Moreover, larger tumor size was a significant risk factor for survival outcomes $(P<0.01)$ (Fig. 1).

\section{Multivariate analysis of prognostic factors}

Multivariate analysis with Cox regression was performed to assess for various prognostic factors. Consistent with univariate analysis, pre-albumin [hazard ratio (HR) 0.512; $95 \%$ CI $0.282-0.927 ; P=0.027]$ and TNM stage (HR 2.532; $95 \%$ CI 1.220-5.523; $P=0.013$ ) were independent prognostic indicators and a high level of prealbumin demonstrated a positive survival. However, the level of hemoglobin, tumor size, and PNI were not a significant independent factor in multivariate analysis $(P>0.05)$ (Table 2).

\section{Definition of prognostic factors}

Considering the interactions of patient-related factors for survival outcomes, ROC curves were constructed to estimate their discrimination ability (Fig. 2). The hemoglobin AUC was 0.617 (95\% CI 0.507-0.727); the pre-albumin AUC was 0.660 (95\% CI 0.553-0.767); and the PNI AUC was 0.623 (95\% CI 0.513-0.733). Therefore, the performance abilities of pre-albumin were similar to others for predicting overall survivals of AEG patients.

\section{Relationship between the pre-albumin and clinicopathologic characteristics}

The serum pre-albumin, as an independent prognostic indicator, was associated with survival outcomes in AEG patients. Thus, subgroup analyses were further established to identify the relationships between the prealbumin and other clinicopathologic characteristics and evaluate the prognostic value of pre-albumin more 

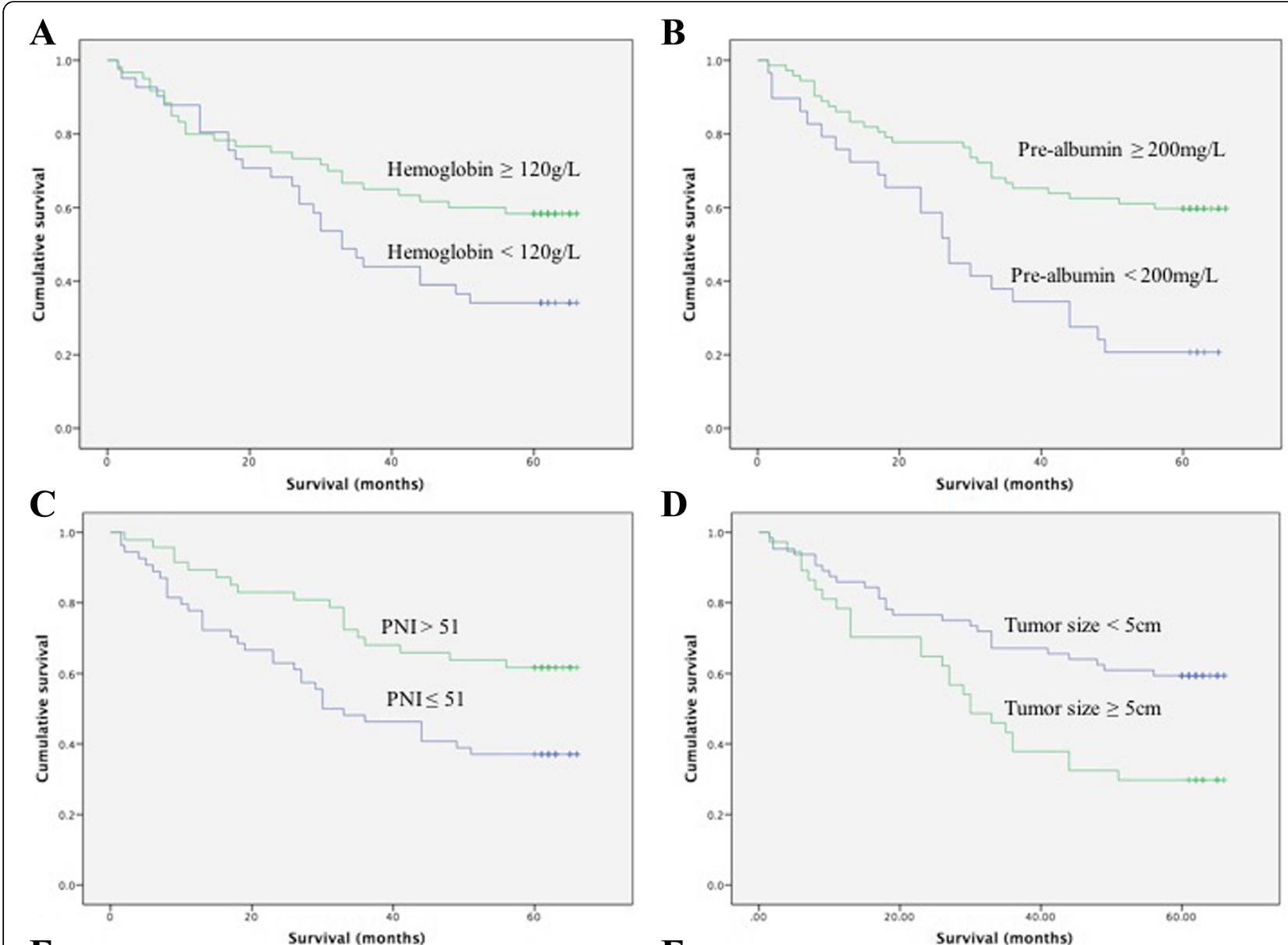

D
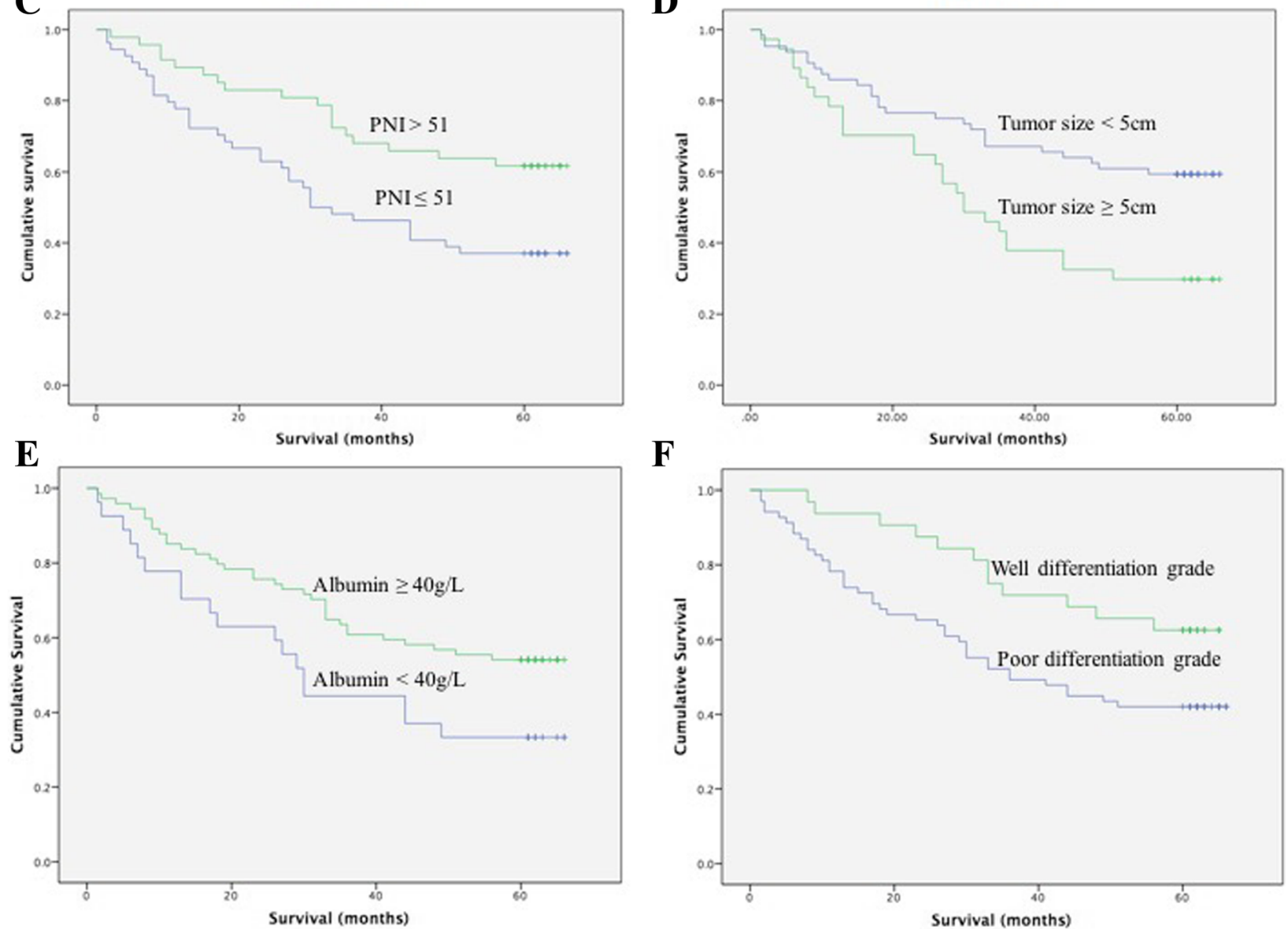

Fig. 1 Kaplan-Meier survival curves after surgery for AEG patients in high hemoglobin and low hemoglobin (a), high pre-albumin and low prealbumin (b), high prognostic nutritional index (PNI) and low PNI (c), large tumor size and small tumor size (d), high albumin and low albumin (e), and well differentiation grade and poor differentiation grade $(\mathbf{f})$

comprehensively (Table 3). Among them, a total of 29 AEG patients were detected with a lower level of prealbumin $<200 \mathrm{mg} / \mathrm{L}$, whereas others were detected with a higher level of pre-albumin $\geq 200 \mathrm{mg} / \mathrm{L}$. The pre-albumin was not significantly correlated with gender, age, BMI, albumin, and tumor location. However, the associations between pre-albumin and hemoglobin $(P=0.014)$, pre-albumin and PNI $(P=0.011)$, and prealbumin and differentiation grade $(P=0.011)$ were significant. More importantly, the level of serum prealbumin was significantly associated with TNM stage $(P<0.001)$. 
Table 2 Multivariate analysis of prognostic factors for OS in AEG patients

\begin{tabular}{|c|c|c|c|}
\hline Patient-related factors & Hazard ratio & $95 \% \mathrm{Cl}$ & $P$ value \\
\hline \multicolumn{4}{|l|}{ Albumin (g/L) } \\
\hline$<40$ & 1 & & 0.874 \\
\hline$\geq 40$ & 0.945 & $0.469-1.903$ & \\
\hline \multicolumn{4}{|l|}{ Hemoglobin (g/L) } \\
\hline$<120$ & 1 & & 1.000 \\
\hline$\geq 120$ & 1.000 & $0.527-1.899$ & \\
\hline \multicolumn{4}{|l|}{ PNI } \\
\hline$<51$ & 1 & & 0.426 \\
\hline$\geq 51$ & 0.751 & $0.372-1.518$ & \\
\hline \multicolumn{4}{|l|}{ Pre-albumin (g/L) } \\
\hline$<200$ & 1 & & $0.021^{*}$ \\
\hline$\geq 200$ & 0.494 & $0.271-0.901$ & \\
\hline \multicolumn{4}{|l|}{ Tumor size (cm) } \\
\hline$<5$ & 1 & & 0.869 \\
\hline$\geq 5$ & 1.053 & $0.567-1.957$ & \\
\hline \multicolumn{4}{|l|}{ TNM stage } \\
\hline l, II & 1 & & $0.013^{*}$ \\
\hline III, IV & 2.530 & $1.220-5.248$ & \\
\hline \multicolumn{4}{|l|}{ Differentiation grade } \\
\hline Poor & 1 & & 0.248 \\
\hline Well & 1.335 & $0.307-1.357$ & \\
\hline
\end{tabular}

$P N I$ prognostic nutritional index ${ }^{*} P<0.05$

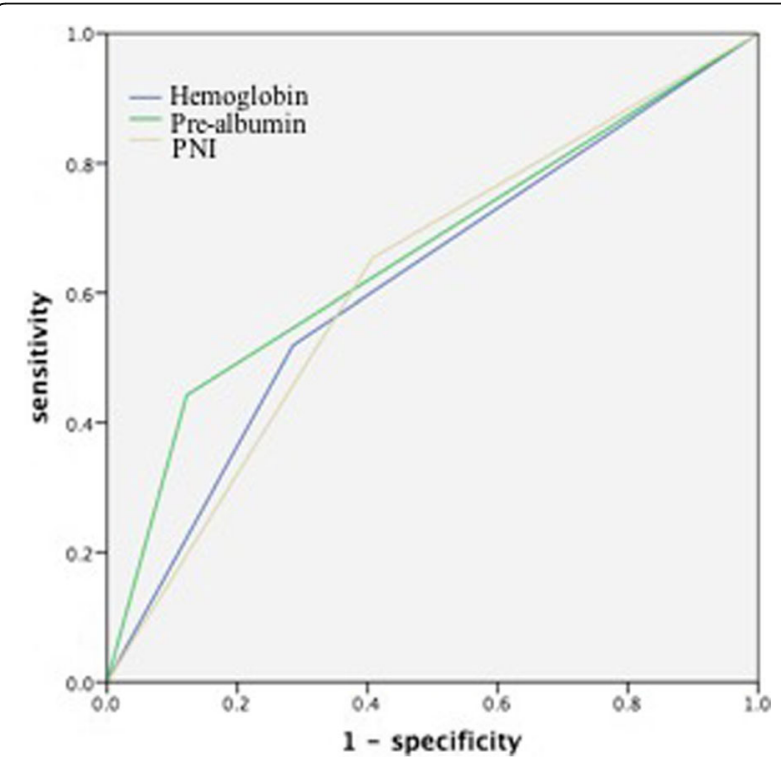

Fig. 2 The predictive ability of the three parameters for 5-year OS was compared by ROC curves (PNI represents prognostic nutritional index)

\section{Discussion}

In the few decades, there has been an alarming rise in the incidence of tumors originating at the esophagogastric junction. In some literature, some authors held the opinion that all tumors arising at or close to the esophagogastric junction should be traditionally classified into esophageal carcinomas or gastric cancers, while Siewert et al. considered them as an entity called AEG and classified them into three types: I-III [2, 15-17]. In Eastern countries, types I and II of AEG were more prevalent than type III, which is in sharp contrast with the prevalence of the three types in Western countries [18]. The AEG-special etiological factors for dramatic increase of prevalence were remaining not determined. Continuous gastroesophageal reflux was reported to increase the risk of epithelium to progress to Barrett's esophagus or adenocarcinoma [19]. Complete removal of primary tumor (R0 resection) with lymphadenectomy (D2), as routine radical surgery, remains the curative treatment that provided best survival outcomes. Nowadays, the differences of the 5-year survival rates (>50\%) were demonstrated to having no obvious significance in subtypes and better than tumors at other locations of GC, which was consistent with the results of this study [20].

Preoperative nutritional status is one of critical factors for patient outcomes in a variety of surgeries, especially in gastrectomy. Currently, pre-albumin became the research focus as a serum biomarker for assessment of nutritional status due to shorter half-life (about 1.9 days) than albumin, which is a negative acute-phase protein synthesized in the liver. Additionally, because of the intense from gastric cancer surgery, acute-phase proteins were synthesized in the liver from structural proteins in the plasma. Therefore, the level of pre-albumin has a high sensitivity for understanding the metabolism state and immunity of the body. This study found that a high level of pre-albumin predicted a longer OS than low level in AEG patients. Moreover, pre-albumin was an independent factor for predicting postoperative survival outcomes. $\mathrm{Li}$ et al. reported that postoperative levels of pre-albumin and high-sensitivity C-reactive protein were associated with short-term outcomes and complications after gastrectomy, especially in elderly patients [21]. Due to absence of complete postoperative pre-albumin for this study, the relationships with surgical intense and short-term outcomes had not been determined further in AEG patients.

Another index for assessing the immune and nutrition status was PNI, which was demonstrated to predicting the survival outcomes in patients after gastric cancer surgery. This study suggested AEG patients with a high level of PNI had a longer OS than others, although it was not an independent predictor in univariate analysis. One study of 548 patients with gastric cancer who 
Table 3 Relationship between the pre-albumin and clinicopathologic characteristics

\begin{tabular}{|c|c|c|c|}
\hline $\begin{array}{l}\text { Patient-related } \\
\text { factors }\end{array}$ & $\begin{array}{l}\text { Pre-albumin } \\
<200 \mathrm{mg} / \mathrm{L} \\
(n=29)\end{array}$ & $\begin{array}{l}\text { Pre-albumin } \\
\geq 200 \mathrm{mg} / \mathrm{L} \\
(n=72)\end{array}$ & $P$ value \\
\hline Gender & & & 0.260 \\
\hline Male & 22 & 58 & \\
\hline Female & 7 & 14 & \\
\hline Age (years) & & & 0.565 \\
\hline$<60$ & 4 & 19 & \\
\hline$\geq 60$ & 25 & 53 & \\
\hline BMI $\left(\mathrm{kg} / \mathrm{m}^{2}\right)$ & & & 0.084 \\
\hline$<18.5$ & 7 & 2 & \\
\hline $18.5 \leq<23$ & 13 & 38 & \\
\hline $23 \leq$ & 9 & 32 & \\
\hline Hemoglobin (g/L) & & & $0.014^{*}$ \\
\hline$<120$ & 17 & 24 & \\
\hline$\geq 120$ & 12 & 48 & \\
\hline Albumin (g/L) & & & 0.052 \\
\hline$<40$ & 14 & 13 & \\
\hline$\geq 40$ & 15 & 59 & \\
\hline $\mathrm{PNI}$ & & & $0.011^{*}$ \\
\hline$<51$ & 20 & 34 & \\
\hline$\geq 51$ & 9 & 38 & \\
\hline Differentiation grade & & & $0.044^{*}$ \\
\hline Poor & 20 & 49 & \\
\hline Well & 9 & 23 & \\
\hline Tumor location & & & 0.632 \\
\hline Siewert II & 1 & 5 & \\
\hline Siewert III & 28 & 67 & \\
\hline Tumor size (cm) & & & $0.004^{*}$ \\
\hline$<5$ & 14 & 50 & \\
\hline$\geq 5$ & 15 & 22 & \\
\hline TNM stage & & & $<0.001^{*}$ \\
\hline I, II & 10 & 34 & \\
\hline III, IV & 19 & 38 & \\
\hline
\end{tabular}

$B M I$ body mass index, $P N /$ prognostic nutritional index ${ }^{*} P<0.05$

underwent gastrectomy found that low PNI was associated with less tumor depth and lymph node metastasis [22]. Currently, the cutoff values of PNI in different studies were not same which were determined by ROC curves or X-title program, so a deterministic value of PNI to assessing survival outcomes is not scientific.

The major limitations of this study were lack of complete specific tumor markers and inflammatory markers and was a retrospective single-center clinical research. Because patients with AEG of type I were treated by the Department of Thoracic Surgery, the subtype analysis had not been completed.

\section{Conclusions}

This study first established the relationships between preoperative indexes of hematology and prognosis of AEG patients and found that AEG patients with a high level of pre-albumin, hemoglobin, and PNI had longer OS. Moreover, our study further demonstrated that preoperative pre-albumin was an independent prognostic factor.

\section{Abbreviations}

AEG: Adenocarcinoma of esophagogastric junction; AJCC: American Joint Committee on Cancer; FAHAMU: First Affiliated Hospital of Anhui Medical University; NLR: Neutrophil to lymphocytes ratio; OS: Overall survival; PNI: Prognostic nutritional index; ROC: Receiver operating characteristic; SPSS: Statistical Package for the Social Sciences; TNM: Tumor-node-metastasis

\section{Acknowledgements}

Wen-Xiu Han and Zhang-Ming Chen contributed equally to this study. The authors wish to thank Prof. Songcheng Ying for his assistance in editing and proofreading the manuscript.

\section{Funding}

This work was supported by the National Science Foundation of China (No.81572350 to A-man Xu).

\section{Availability of data and materials}

The dataset supporting the conclusions of this article is available upon request.

\section{Authors' contributions}

AMX conceived and designed the experiments. WXH and ZMC collected and analyzed the data. AMX, WXH, and ZMC wrote the manuscript. ZJW helped to identify the results of the statistical analysis. All authors read and approved the final manuscript.

\section{Competing interests}

The authors declare that they have no competing interests.

\section{Consent for publication}

Not applicable.

\section{Ethics approval and consent to participate}

This study has been performed in accordance with the Declaration of Helsinki. The research protocol was approved by the First Affiliated Hospital of Anhui Medical University Ethic committee. All patients reviewed this research and signed patient informed consent.

Received: 13 February 2016 Accepted: 25 October 2016 Published online: 03 November 2016

\section{References}

1. Naghavi M. The global burden of cancer. JAMA Oncol. 2015;1:505-27.

2. Siewert. Adenocarcinoma of the esophago-gastric junction. Gastric Cancer. 1999:2:87-8.

3. Steevens J, Botterweck AA, Dirx MJ, van den Brandt PA, Schouten LJ. Trends in incidence of oesophageal and stomach cancer subtypes in Europe. Eur J Gastroenterol Hepatol. 2010;22:669-78

4. Deans C, Yeo MS, Soe MY, Shabbir A, Ti TK, So JB. Cancer of the gastric cardia is rising in incidence in an Asian population and is associated with adverse outcome. World J Surg. 2011;35:617-24.

5. Kusano C, Gotoda T, Khor CJ, Katai H, Kato H, Taniguchi H, Shimoda T. Changing trends in the proportion of adenocarcinoma of the esophagogastric junction in a large tertiary referral center in Japan. J Gastroenterol Hepatol. 2008:23:1662-5.

6. Siewert JR, Stein HJ, Feith M. Adenocarcinoma of esophago-gastric junction. Scand J Surg. 2006;95:260-9. 
7. Stein $H J$, Feith M, Siewert JR. Cancer of the esophagogastric junction. Surg Oncol. 2000;9:35-41.

8. Hu B, Yang XR, Xu Y, Sun YF, Sun C, Guo W, et al. Systemic immuneinflammation index predicts prognosis of patients after curative resection for hepatocellular carcinoma. Clin Cancer Res. 2014;20:6212-22.

9. Walsh SR, Cook EJ, Goulder F, Justin TA, Keeling NJ. Neutrophil-lymphocyte ratio as a prognostic factor in colorectal cancer. J Surg Oncol. 2005;91:181-4.

10. Azab B, Bhatt VR, Phookan J, Murukutla S, Kohn N, Terjanian, et al. Usefulness of the neutrophil-to-lymphocyte ratio in predicting short- and long-term mortality in breast cancer patients. Ann Surg Oncol. 2012;19:217-24.

11. Caro JJ, Salas M, Ward A, Goss G. Anemia as an independent prognostic factor for survival in patients with cancer: a systemic, quantitative review. Cancer. 2001;91:2214-21.

12. Washington $\mathrm{K}$. 7th edition of the AJCC cancer staging manual: stomach. Ann Surg Oncol. 2010:17:3077-9.

13. Onodera T, Goseki N, Kosaki G. Prognostic nutritional index in gastrointestinal surgery of malnourished cancer patients. Nihon Geka Gakkai Zasshi. 1984;85:1001-5.

14. Youden WJ. Index for rating diagnostic tests. Cancer. 1950;3:32-5.

15. DeMeester SR. Adenocarcinoma of the esophagus and cardia: a review of the disease and its treatment. Ann Surg oncol. 2006;13:12-30.

16. Hennessy TPJ, Keeling P. Adenocarcinoma of the esophagus and cardia. J Thorac Cardiovasc Surg. 1987;94:64-8.

17. Husemann B. Cardia carcinoma considered as a distinct clinical entity. Br J Surg. 1989;76:136-9.

18. Hasegawa S, Yoshikawa T, Cho H, Tsuburaya A, Kobayashi O. Is adenocarcinoma of the esophagogastric junction difference between Japan and Western countries? The incidence and clinicopathological features at a Japanese high-volume cancer center. World J Surg. 2009;33:95-103.

19. Hirota WK, Loughney TM, Lazas DJ, Maydonovitch CL, Rholl V, Wong RK. Specialized intestinal metaplasia, dysplasia, and cancer of the esophagus and esophagogastric junction: prevalence and clinical data. Gastroenterology. 1999;116:277-85.

20. Hosokawa Y. Clinicopathological features and prognostic factors of adenocarcinoma of the esophagogastric junction according to Siewert classification: Experiences at a single institution in Japan. Ann Surg Oncol. 2012;19:677-83

21. Li B, Liu HY, Guo SH, Sun P, Gong FM, Jia BQ. Impact of early enteral and parenteral nutrition on prealbumin and high-sensitivity C-reactive protein after gastric surgery. Genet Mol Res. 2015;14:7130-5.

22. Migita K, Takayama T, Saeki K, Matsumoto S, Wakatsuki K, Enomoto K, et al. The prognostic nutritional index predicts long-term outcomes of gastric cancer patients independent of tumor stage. Ann Surg Oncol. 2013;20: 2647-54.

\section{Submit your next manuscript to BioMed Central and we will help you at every step:}

- We accept pre-submission inquiries

- Our selector tool helps you to find the most relevant journal

- We provide round the clock customer support

- Convenient online submission

- Thorough peer review

- Inclusion in PubMed and all major indexing services

- Maximum visibility for your research

Submit your manuscript at www.biomedcentral.com/submit

CBiomed Central 